\title{
The Medicago truncatula Yellow Stripe1-Like3 gene is involved in vascular delivery of transition metals to root nodules
}

Rosario Castro-Rodríguez ${ }^{1, *, \dagger}$, Isidro Abreu ${ }^{1, *, \neq}$, María Reguera $^{1}$, Lorena Novoa-Aponte ${ }^{2}$, Ana Mijovilovich ${ }^{3}$, Viviana Escudero', Francisco J. Jiménez-Pastor ${ }^{4}$, Javier Abadía $^{4}$, Jiangqi Wen ${ }^{5}$, Kirankumar S. Mysore ${ }^{5}$, Ana Álvarez-Fernández ${ }^{4}$, Hendrik Küpper ${ }^{3,6}$, Juan Imperial ${ }^{7}$ and Manuel González-Guerrero ${ }^{1,8, \S}$

${ }^{1}$ Centro de Biotecnología y Genómica de Plantas (UPM-INIA), Universidad Politécnica de Madrid, Campus de Montegancedo, Crta. M-40 km 38, 28223 Pozuelo de Alarcón (Madrid), Spain

${ }^{2}$ Department of Chemistry and Biochemistry, Worcester Polytechnic Institute, 100 Institute Road, Worcester, MA 01609, USA

${ }^{3}$ Czech Academy of Sciences, Biology Centre, Institute of Plant Molecular Biology, Department of Plant Biophysics and Biochemistry, CZ-37005 Česke Budějovice, Czech Republic

${ }^{4}$ Estación Experimental de Aula Dei, Consejo Superior de Investigaciones Científicas (EEAD-CSIC), Avda. Montañana 1005, 50059 Zaragoza, Spain

${ }^{5}$ Noble Research Institute, Ardmore, OK 73401. USA

${ }^{6}$ University of South Bohemia, Department of Experimental Plant Biology, Branišovská 31/1160, 37005 Ceské Budějovice, Czech Republic

7 Instituto de Ciencias Agrarias, Consejo Superior de Investigaciones Científicas (ICA-CSIC), Serrano, 115 bis, 28006 Madrid, Spain

${ }^{8}$ Escuela Técnica Superior de Ingeniería Agronómica, Alimentaria y de Biosistemas, Universidad Politécnica de Madrid, 28040 Madrid, Spain

† Present address: Department of Biology. University of Massachusetts. Amherst, MA 01003, USA.

‡ Present address: Department of Plant Sciences. University of Oxford, Oxford OX1 3RB, UK.

* These authors contributed equally to this work.

§ Correspondence: manuel.gonzalez@upm.es

Received 18 August 2020; Accepted 18 August 2020

Editor: Miriam Gifford, University of Warwick, UK

\begin{abstract}
Symbiotic nitrogen fixation carried out in legume root nodules requires transition metals. These nutrients are delivered by the host plant to the endosymbiotic nitrogen-fixing bacteria living within the nodule cells, a process in which vascular transport is essential. As members of the Yellow Stripe-Like (YSL) family of metal transporters are involved in root to shoot transport, they should also be required for root to nodule metal delivery. The genome of the model legume Medicago truncatula encodes eight YSL proteins, four of them with a high degree of similarity to Arabidopsis thaliana YSLs involved in long-distance metal trafficking. Among them, MtYSL3 is a plasma membrane protein expressed by vascular cells in roots and nodules and by cortical nodule cells. Reducing the expression level of this gene had no major effect on plant physiology when assimilable nitrogen was provided in the nutrient solution. However, nodule functioning was severely impaired, with a significant reduction of nitrogen fixation capabilities. Further, iron and zinc accumulation and distribution changed. Iron was retained in the apical region of the nodule, while zinc became strongly accumulated in the nodule veins in the ys/3 mutant. These data suggest a role for MtYSL3 in vascular delivery of iron and zinc to symbiotic nitrogen fixation.
\end{abstract}

Keywords: Iron, Medicago, micro-X-ray fluorescence ( $\mu$ XRF), nitrogenase, symbiotic nitrogen fixation, transition metal transport.

(C) The Author(s) 2020. Published by Oxford University Press on behalf of the Society for Experimental Biology. All rights reserved. For permissions, please email: journals.permissions@oup.com 


\section{Introduction}

Iron, copper, and other transition metals are required at relatively high levels for biological nitrogen fixation, the conversion of $\mathrm{N}_{2}$ into $\mathrm{NH}_{4}^{+}$carried out by diazotrophic microorganisms (González-Guerrero et al., 2014, 2016). These elements act as cofactors of key enzymes mediating the process, such as nitrogenases that directly catalyse the reaction, cytochrome oxidases that provide energy to the reaction and control $\mathrm{O}_{2}$ levels, and many of the free radical detoxification enzymes (Appleby, 1984; Rubio et al., 2004; Rubio and Ludden, 2005). Therefore, ensuring proper transition metal uptake is critical for any diazotrophic organism. Free-living nitrogen-fixing bacteria use a large battery of siderophores, transition metal transporters, and storage proteins to directly acquire these metals from the environment (Jurkevitch et al., 1992; Yeoman et al., 2000; Navarro-Rodríguez et al., 2019). In contrast, symbiotic diazotrophs must obtain the required metal nutrients through their host (González-Guerrero et al., 2016).

The paradigmatic example of a symbiotic diazotroph are the bacteria known as rhizobia. These organisms colonize the cells of legume root nodules, organs developed to provide the conditions for nitrogen fixation to occur (Downie, 2014). Within the nodule cells, rhizobia are surrounded by plant-derived membranes, the symbiosome membranes, and differentiate into the nitrogen-fixing form, the bacteroids (Kereszt et al., 2011). Across the symbiosome membrane, bacteroids deliver the fixed nitrogen while they receive photosynthates, phosphate, sulfur, as well as the essential transition elements needed for nitrogen fixation (Udvardi and Poole, 2013). Transition metal nutrients are delivered from the plant root to the nodule through the vasculature and released in the apoplast of the area of bacteroid differentiation (Rodríguez-Haas et al., 2013), in a process that resembles metal delivery to shoots (Conte and Walker, 2011). There, different metal transporters introduce these nutrients into the nodule cell cytosol and transfer them across the symbiosome membranes. Several of them have been identified, particularly those proteins located at the host cell plasma membrane and at the symbiosome membrane (TejadaJiménez et al., 2015, 2017; Abreu et al., 2017; Senovilla et al., 2018; Brear et al., 2020; Escudero et al., 2020b; Walton et al., 2020). However, it largely remains to be determined how vascular transport occurs and which proteins are mediating it.

Transition metal loading in the root vasculature is mediated by transporters of the ferroportin and $\mathrm{P}_{1 \mathrm{~b}}$-ATPase families (Hussain et al., 2004; Andrés-Colás et al., 2006; Morrissey et al., 2009). Once in the saps, metals are chelated by a collection of soluble molecules, with a prominent role for citrate and nicotianamine (NA) (Flis et al., 2016). These molecules facilitate metal solubility and prevent oxidative damage. When metals reach the shoots, they are recovered from the sap as metal-NA complexes and introduced into the cells by members of the Yellow Stripe-Like (YSL) family (Curie et al., 2009). These are a family of plant-specific proteins participating in remobilization of intracellular metal reserves, mediating long-distance metal trafficking and signalling, and in metal uptake from soil by grasses (Curie et al., 2001; Waters et al., 2006; Conte et al., 2013; Kumar et al., 2017). Together with the oligopeptide transporters (OPTs), YSLs are included in the oligopeptide transport superfamily (Lubkowitz, 2011). In Arabidopsis thaliana, AtYSL1 and AtYSL3 are responsible for iron delivery to shoots as well as for signalling iron sufficiency (Waters et al., 2006; Kumar et al., 2017). Considering the high metal demand of nitrogen fixation (O'Hara, 2001), a large portion of these nutrients has to be delivered to nodules, where similar mechanisms to those reported in shoots would probably be in place. Therefore, it should be expected that metal-NA-transportingYSLs similar to AtYSL1 or AtYSL3 are functional in the nodule vasculature. Recent identification of nodule nicotianamine synthases and evidence of their importance for iron homeostasis in nodules supports this hypothesis (Avenhaus et al., 2016; Escudero et al., 2020a).

Here we report the role of Medtr3g092090, MtYSL3, a Medicago truncatula orthologue of AtYSL3, highly expressed in nodules and with a vascular localization. Mutation of MtYSL3 results in a reduction of nitrogenase activity that affects plant growth, the likely consequence of reduced iron and zinc content in nodules and its altered distribution. The data are consistent with a role for MtYSL3 in iron and zinc delivery to nodules.

\section{Materials and methods}

\section{Biological materials and plant growth conditions}

Medicago truncatula seeds were scarified and surface sterilized following the same protocol described in Tejada-Jiménez et al. (2015). After a previous pre-germination step in $0.8 \%$ water agar plates during $48 \mathrm{~h}$ at $22{ }^{\circ} \mathrm{C}$, seedlings were planted in sterilized perlite pots and inoculated with Sinorhizobium meliloti 2011 or S. meliloti 2011 transformed with pHC60 [green fluorescent protein (GFP)-expressing vector] (Cheng and Walker, 1998) for nodulation assays. Nodules were collected 28 days postinoculation (dpi). For non-symbiotic experiments, plants were watered every 2 weeks with solutions supplemented with $2 \mathrm{mM} \mathrm{NH}_{4} \mathrm{NO}_{3}$. In all cases, plants were watered every $2 \mathrm{~d}$ alternating Jenner's solution with water (Brito et al., 1994) and they were grown in a greenhouse under $16 \mathrm{~h}$ light $/ 8 \mathrm{~h}$ dark at $25{ }^{\circ} \mathrm{C} / 20{ }^{\circ} \mathrm{C}$. When required, the nutrient solution was fortified with $38 \mu \mathrm{M}$ zinc or $0.5 \mathrm{~g} \mathrm{l}^{-1} \mathrm{Fe}$ (III)-EDDHA [ethylendiaminedi(o-hydroxyphenylacetate)]. For hairy root transformations, $M$. truncatula seedlings were transformed with Agrobacterium rhizogenes ARqua1 carrying the appropriate binary vector, as previously described (Boisson-Dernier et al., 2001). Transient expression in Nicotiana benthamiana was performed by transforming leaves with the plasmid constructs in Agrobacterium tumefaciens C58C1 (Deblaere et al., 1985). These plants were grown in the greenhouse under the same conditions as for M. truncatula.

\section{RNA isolation and quantitative real-time PCR}

RNA was extracted from shoots, roots, and nodules using Tri-Reagent (Life Technologies), treated with DNase, and cleaned with the RNeasy Minikit (Qiagen,Valencia, CA, USA). A $1 \mu \mathrm{g}$ aliquot of DNA-free RNA was used to synthesize cDNA by using a PrimeScript ${ }^{\mathrm{TM}}$ RT Reagent Kit (TAKARA, Kusatsu, Shiga, Japan).

Gene expression was studied by quantitative real-time PCR (StepOne plus, Applied Biosystems) using the Power SyBR Green master mix (Applied Biosystems). Primers used are listed in Supplementary Table S1 at $J X B$ online. RNA levels were normalized by using the ubiquitin carboxyterminal hydrolase gene as internal standard for M. truncatula genes (Kakar et al., 2008). 


\section{$\beta$-Glucuronidase (GUS) assay}

A transcriptional fusion between the MtYSL3 promoter and the GUS gene was constructed by amplifying $2 \mathrm{~kb}$ upstream of the MtYSL3 start codon using the primers indicated in Supplementary Table S1. This amplicon was inserted into pDONR207 and transferred to pGWB3 (Nakagawa et al., 2007) using Gateway technology ${ }^{\circledR}$ (Invitrogen). Medicago truncatula R108 roots were transformed as indicated above. Transformed plants were transferred to sterilized perlite pots and inoculated with S. meliloti 2011. GUS activity was determined in 28 dpi plants as described (Vernoud et al., 1999).

\section{Immunolocalization of MtYSL3-HA}

The genomic full sequence of MtYSL3 including $2 \mathrm{~kb}$ upstream of its start codon was amplified using the primers indicated in Supplementary Table S1 and fused with three C-terminal haemagglutinin (HA) epitopes in-frame by cloning into pGWB13 (Nakagawa et al., 2007). Hairy root transformation was performed as described previously by Boisson-Dernier et al. (2001). Transformed plants were inoculated with S. meliloti 2011 containing the pHC60 plasmid that constitutively expresses GFP. After 28 dpi, nodules and roots were collected. Fixation and immunohistochemistry protocols were carried out as indicated in TejadaJiménez et al. (2015).

\section{Transient expression in N. benthamiana leaves}

The MtYSL3 coding sequence was cloned into pGWB6 (Nakagawa et al., 2007) using Gateway Technology (Invitrogen) resulting in an N-terminal fusion with GFP.This construct and the plasma membrane marker $\mathrm{pBIN}$ AtPIP2-CFP (cyan fluorescent protein; Nelson et al., 2007) were introduced into $A$. tumefaciens C58C1 (Deblaere et al., 1985). Transformants were grown in a liquid medium to late exponential phase, centrifuged, and resuspended to an $\mathrm{OD}_{600}$ of 1.0 in $10 \mathrm{mM}$ MES pH 5.6, containing $10 \mathrm{mM} \mathrm{MgCl}_{2}$ and $150 \mu \mathrm{M}$ acetosyringone. These cells were mixed with an equal volume of $A$. tumefaciens C58C1 expressing the silencing suppressor p19 of Tomato bushy stunt virus (pCH32 35S:p19) (Wood et al., 2009). Bacterial suspensions were incubated for $3 \mathrm{~h}$ at room temperature and then injected into young leaves of 4-week-old N. benthamiana plants. Expression of the appropriate construct was analysed after $3 \mathrm{~d}$ by confocal laser-scanning microscopy (Leica SP8) with excitation at $405 \mathrm{~nm}$ for CFP and $488 \mathrm{~nm}$ for GFP.

\section{Nitrogenase activity}

The acetylene reduction assay was used to measure the nitrogenase activity (Hardy et al., 1968). Wild-type and mutant roots at 28 dpi were introduced separately in $30 \mathrm{ml}$ tubes fitted with rubber stoppers. Each tube contained between three and five roots. A $3 \mathrm{ml}$ volume of air in each tube was replaced by the same volume of acetylene and subsequently the tubes were incubated for $30 \mathrm{~min}$ at room temperature. Gas samples $(0.5 \mathrm{ml})$ were analysed in a Shimadzu GC-8A gas chromatograph fitted with a Porapak $\mathrm{N}$ column. The amount of ethylene produced was determined by measuring the height of the ethylene peak relative to background. Each point consists of two tubes each measured in triplicate. After measurements, nodules were recovered from roots to measure their weight.

\section{Metal content measurements}

Iron, copper, and zinc content were determined in shoots, roots, and nodules at 28 dpi. Plant tissues were weighed and mineralized in 15.6 $\mathrm{M} \mathrm{HNO}_{3}$ (trace metal grade) for $1 \mathrm{~h}$ at $80{ }^{\circ} \mathrm{C}$ and overnight at $20^{\circ} \mathrm{C}$. Digestions were completed with $2 \mathrm{M} \mathrm{H}_{2} \mathrm{O}_{2}$. Samples were diluted in $300 \mathrm{mM} \mathrm{HNO}_{3}$ prior to measurements. Element analyses were performed with atomic absorption spectroscopy in an AAnalyst 800 (Perkin Elmer), equipped with a graphite furnace. All samples were measured in duplicate.

\section{Metal localization by micro-X-ray fluorescence ( $\mu$ XRF)}

A customized benchtop $\mu$ XRF beamline 'M4 Tornado' (Bruker Nano $\mathrm{GmbH}$, Germany), as described in detail by Mijovilovich et al. (2020), was used for analysing tissue-level metal distribution in the root nodules. In brief, in this machine the nodules were kept alive in a custom-designed measuring chamber where throughout the measurement they were kept in Jenner's solution. The measurement was done by excitation with a Rh tube with fibre optic focusing of the beam to $15 \mu \mathrm{m}$ and filtering of the excitation spectrum with an AlTi filter. A step size of $8 \mu \mathrm{m}$ was applied to yield a $2 \times$ oversampling. The measured $\mu \mathrm{XRF}$ spectra in each pixel of the $\mu \mathrm{XRF}$ maps were deconvoluted using the software supplied with the Tornado. The net counts in the resulting element distribution maps were recalculated to millimolar concentrations according to a certified liquid standard (standard solution VI, Merck KGaA Darmstadt Germany) in a cuvette of the thickness of an average nodule. Colour scales were assigned to the quantified data using ImageJ, after which they were converted from 16 bit to RGB format for assembling the figure using PhotoImpact X3 (Corel Corporation, Ottawa, Canada).

\section{NA content determination}

NA was extracted as previously described (Banakar et al., 2017) with some modifications. Briefly, NA was extracted from $\sim 50 \mathrm{mg}$ of nodules, and frozen then ground in $400 \mu \mathrm{l}$ of milliQ water spiked with nicotyl-lysine $(150 \mu \mathrm{M}$ final concentration) as internal standard. Samples were homogenized in a mixer mill (Retsch MM300, Retsch) during $5 \mathrm{~min}$ at $30 \mathrm{~s}^{-1}$ frequency, and then centrifuged at $12000 \mathrm{~g}$ for $10 \mathrm{~min}$ at $4{ }^{\circ} \mathrm{C}$. The supernatant was then passed through a $3 \mathrm{kDa}$ cut-off centrifugal filter (cellulose Amicon ${ }^{\circledR}$, Merck) $(1 \mathrm{~h}$ at 14000 $g$ at $\left.4{ }^{\circ} \mathrm{C}\right)$ and dried under vacuum $\left(1.5 \mathrm{~h}\right.$ at $\left.40^{\circ} \mathrm{C}\right)$. Dry residues from shoots were dissolved in $20 \mu \mathrm{l}$ of milliQ water, whereas root and nodule dry residues were dissolved in $15 \mu \mathrm{l}$. Then $5 \mu \mathrm{l}$ aliquots were mixed with EDTA (final concentration $8.33 \mathrm{mM}$ ) to dissociate potential NA-metal complexes, and 50\% (v/v) mobile phase A (see below) to favour chromatographic separation. The mixture was filtered through 0.45 polyvinylidene fluoride (PVDF) ultrafree-MC centrifugal filter devices (Merck) before analysis.

NA levels were determined by HPLC-electrospray ionization time-of-flight MS (HPLC-ESI-TOF-MS) as described by Banakar et al. (2017). The freshly prepared samples were fractionated using an Alliance 2795 HPLC system (Waters) and $\mu \mathrm{LC}$ column (SeQuant ZIC®-HILIC, $15 \mathrm{~cm} \times 1 \mathrm{~mm}$ internal diameter, $5 \mu \mathrm{m}, 200 \AA$, Merck), with a mobile phase consisting of solvent A (10\% $10 \mathrm{mM}$ ammonium acetate $\mathrm{pH} 7.3,90 \%$ acetonitrile) and solvent $\mathrm{B}(80 \% 30 \mathrm{mM}$ ammonium acetate $\mathrm{pH} 7.3,20 \%$ acetonitrile) at a flow rate of $0.15 \mathrm{ml} \mathrm{min}$. The gradient program started at $100 \%$ (v/v) solvent A for 3 min, then decreased linearly to $30 \%(\mathrm{v} / \mathrm{v})$ solvent A over the next $7 \mathrm{~min}$, remained for $7 \mathrm{~min}$ at $30 \%(\mathrm{v} / \mathrm{v})$ solvent $\mathrm{A}$, and then returned to the initial conditions over the next $8 \mathrm{~min}$. The column was then allowed to stabilize for $10 \mathrm{~min}$ at the initial conditions before proceeding to the next injection. The total HPLC run time was $35 \mathrm{~min}$, the injection volume was $10 \mu \mathrm{l}$, and the auto sampler and column temperatures were $6{ }^{\circ} \mathrm{C}$ and $30^{\circ} \mathrm{C}$, respectively. The HPLC was coupled to the MicrOTOF mass spectrometer (Bruker Daltonics) equipped with an ESI source. The operating conditions were optimized by the direct injection of $100 \mu \mathrm{M}$ solutions of NA standard at a flow rate of $180 \mu \mathrm{l} \mathrm{h}^{-1}$. Mass spectra were acquired in negative ion mode over the 150-700 mass-to-charge $(\mathrm{m} / \mathrm{z})$ ratio range. The mass axis was calibrated externally using $\mathrm{Li}$-formate adducts $[10 \mathrm{mM} \mathrm{LiOH}, 0.2 \%$ (v/v) formic acid, and $50 \%(\mathrm{v} / \mathrm{v}) 2$-propanol]. Bruker Daltonik software packages micrOTOF Control v2.2, HyStar v3.2, and Data Analysis v4.0 were used to control the MS, the HPLC interface, and for data processing, respectively. An NA (Toronto Research Chemicals) calibration curve was prepared with nycotyl-lysine as internal standard.

\section{Nodule development studies}

Nodule sections were obtained from 28 dpi nodules from wild-type and mutant plants. The nodules were collected and fixed in $0.25 \%$ 
glutaraldehyde, $4 \%$ formaldehyde, $2.5 \%$ sucrose in $50 \mathrm{mM}$ potassium phosphate buffer ( $\mathrm{pH} 7.4)$ at $4{ }^{\circ} \mathrm{C}$. Samples were dehydrated in an ethanol series and embedded in LR-White resin (London Resin Company Ltd, UK). Finally, nodules were placed in gelatin capsules, filled with resin, and polymerized at $60^{\circ} \mathrm{C}$ for $24 \mathrm{~h}$. Serial thin sections $(0.5 \mu \mathrm{m})$ were cut with a Reichert Ultracut microtome (Leica,Vienna, Austria) fitted with a glass knife at the National Center for Electron Microscopy (Universidad Complutense de Madrid, Spain). Sections were stained with $1 \%$ toluidine blue. Direct observation of sections was performed under a Zeiss Axiophot photomicroscope (Carl Zeiss, Oberkoche, Germany) with an attached digital camera (Leica DFC 420C, Heerburgg, Switzerland).

Nodulation kinetics were determined in $M$. truncatula plate cultures. Sterile seedlings were transferred to plates with Fahreus agar medium (Sauviac et al., 2005) and inoculated with $100 \mu \mathrm{l}$ of saturated culture of S. meliloti. These plants were grown for $28 \mathrm{~d}$ at $16 \mathrm{~h}$ light and $22^{\circ} \mathrm{C}$ and $8 \mathrm{~h}$ darkness and $16^{\circ} \mathrm{C}$. At the indicated time points, nodules per plant were determined, and photographs were taken from representative plants during their development.

\section{Bioinformatics}

To identify $M$. truncatulaYSL family members, BLASTN and BLASTX searches were carried out in the $M$. truncatula genome at Phytozome (https://phytozome.jgi.doe.gov/pz/portal.html). Protein sequences for tree construction were also obtained from Phytozome, as well as from Uniprot (http://www.uniprot.org/blast/), and NCBI (https:// blast.ncbi.nlm.nih.gov/Blast.cgi?PAGE=Proteins): $M . \quad$ truncatula MtYSL1 (Medtr1g077840), MtYSL2 (Medtr1g007540),MtYSL3 (Medtr3g092090), MtYSL4 (Medtr1g007580); A. thaliana AtYSL1 (At4g24120), AtYSL2 (At5g24380), AtYSL3 (At5g53550); Oryza sativa OsYSL15 (Os02g0650300), OsYSL16 (Os04g0542800); Zea mays ZmYS1 (Zm00001d017429), ZmYSL2 (Zm00001d025977); and Brachypodium distachyon BdYS1A (BRADI_3g50267), BdYS1B (BRADI_3g50263), BdYSL2 (BRADI_3g50260), and BdYSL3 (BRADI_5g17230).

Trees were constructed from a ClustalW multiple alignment of the sequences (http://www.ebi.ac.uk/Tools/msa/clustalw2), then analysed by MEGA7 (Kumar et al., 2016) using a Neighbor-Joining algorithm with bootstrapping (1000 iterations). Unrooted trees were visualized with FigTree (http://tree.bio.ed.ac.uk/software/figtree).

Topology modelling was performed using the visualization software PROTTER (http://wlab.ethz.ch/protter/start/), which includes the transmembrane region prediction software Phobius.

\section{Statistical tests}

Data were analysed with Student's unpaired $t$-test to calculate the statistical significance of observed differences. Test results with $P$-values $<0.05$ were considered as statistically significant.

\section{Results}

\section{MtYSL3 is highly expressed in the nodule vasculature}

The proteins of the YSL family cluster in four groups, with group I being the best characterized (Yordem et al., 2011). It includes AtYSL1, 2, and 3, founding protein Zea mays YS1, and four $M$. truncatula YSLs (MtYSL1-MtYSL4, Medtr1g077840, Medtr1g007540, Medtr3g092090, and Medtr1g007580, respectively) (Fig. 1A).AtYSL3 is the closest orthologue to MtYSL3, with $76.6 \%$ identity (AtYSL1 and AtYSL2 had $64.0 \%$ and $72.5 \%$ identity, respectively). Among the M. truncatula group I YSLs, expression of MtYSL4 was not detected in any of the organs tested (Supplementary Fig. S1), while the other three were expressed in shoots and roots from inoculated and non-inoculated plants, as well as in nodules. (Fig. 1B; Supplementary Fig. S1). MtYSL1 transcripts were more abundant in shoots than anywhere else in the plants. MtYSL2 and MtYSL3 transcription was more intense in nodules, the latter being the most highly expressed, approximately four times higher in nodules than in any other plant organ. The inoculation with $S$. meliloti did not result in significant transcriptional changes in shoots or roots compared with non-inoculated nitrogen-fertilized plants. No changes in the expression of MtYSL3 in roots were observed at any time point after inoculation, whereas the expression was higher in nodules at 28 dpi than at $21 \mathrm{dpi}$ (Supplementary Fig. S2).

To locate the tissue expression of MtYSL3, the $2 \mathrm{~kb}$ region upstream of MtYSL3 was used to drive the expression of the gus gene. After $28 \mathrm{dpi}$, roots and nodules were incubated with X-gluc to visualize the GUS activity. The staining pattern was consistent with a vascular expression of MtYSL3 in both organs (Fig. 1C; Supplementary Fig. S3). Longitudinal sections of those nodules revealed a peripheral distribution of the signal, associated with the vasculature, and no expression in the inner nodule region (Fig. 1D). This was also supported by nodule cross-section images (Fig. 1E). In addition, some GUS activity was observed in these sections in cortical nodule cells, although at much lower intensity than in the vasculature. In roots, MtYSL3 expression was confined to the endodermis and inner vascular layers (Fig. 1F).

Immunolocalization of epitope-tagged MtYSL3 supports the results of the GUS activity assays. Three HA tags were fused to the C-terminus of the protein and it was expressed under its own promoter region. MtYSL3-HA localization was visualized using a primary anti-HA mouse antibody and an Alexa594-conjugated anti-mouse antibody. The transformed plants were inoculated with a strain of $S$. meliloti that constitutively expresses GFP. The HA epitope of MtYSL3-HA was detected in the vasculature of the nodule and in cortical cells (Fig. 2A, B). Closer detail of the vascular region showed co-localization with the autofluorescence pattern of the Casparian strip, indicating that MtYSL3-HA was located in the endodermis (Fig. 2C; Supplementary Fig. S4). In roots, MtYSL3-HA was observed in the endodermis and in inner vascular cells, very probably the xylem parenchyma (Fig. 2D). The peripheral distribution of the Alexa594 signal was indicative of a plasma membrane distribution. To test this possibility, $N$. benthamiana leaves were co-agroinfiltrated with a constitutive plasmid expressing MtYSL3 fused to GFP and the plasma membrane marker AtPIP2 fused to CFP. As shown in Fig. 2E, both the GFP and the CFP signal co-localized. Controls did not show any autofluorescence in the Alexa594 channel in the conditions tested (Supplementary Fig. S5). The antibody used did not recognize any native $M$. truncatula nodule protein when incubated with nodules that do not express MtYSL3-HA (Supplementary Fig. S6). 


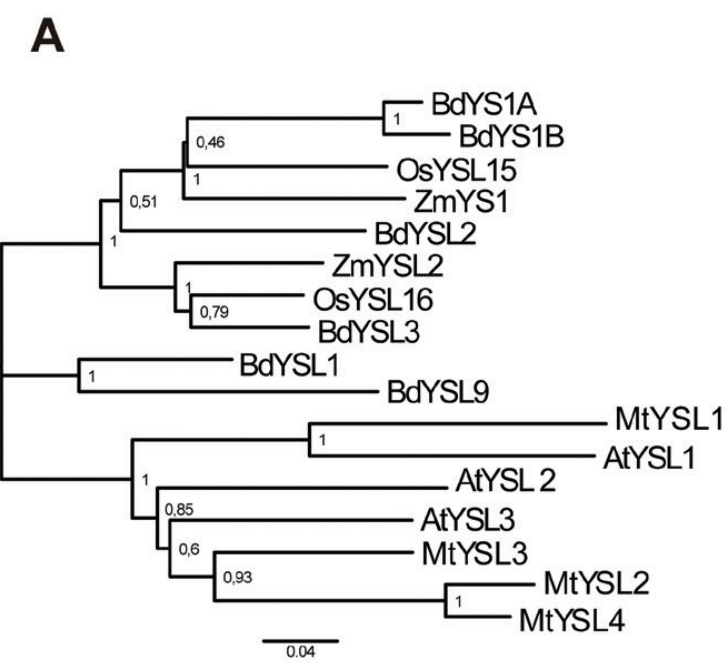

C

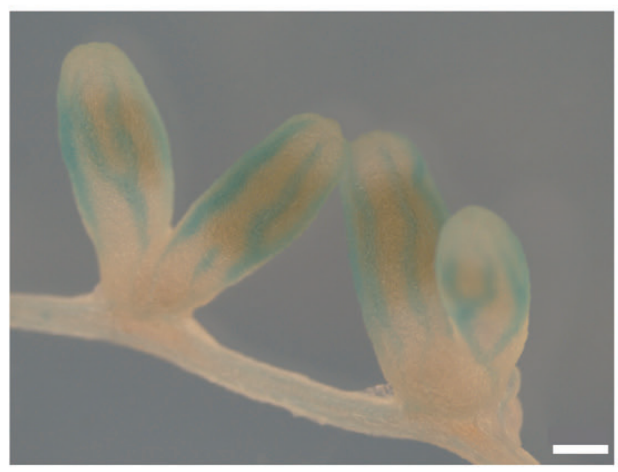

$\mathbf{E}$

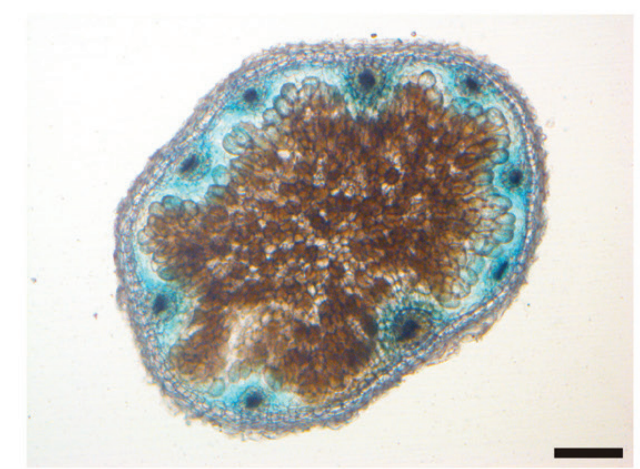

B

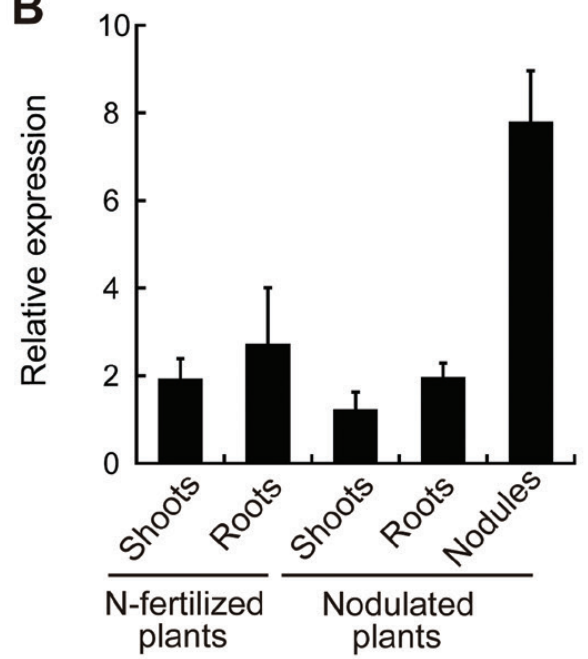

D

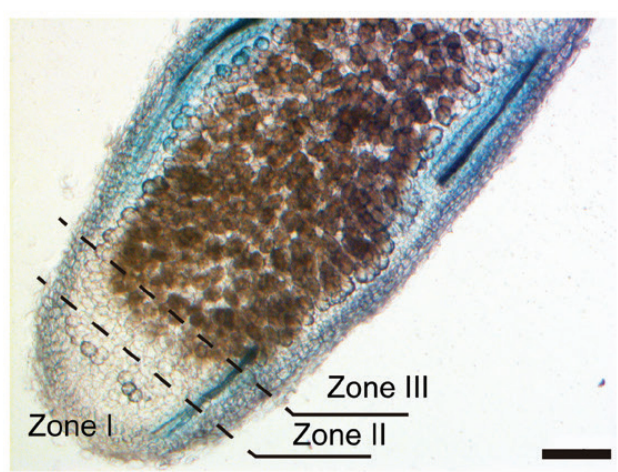

$\mathbf{F}$

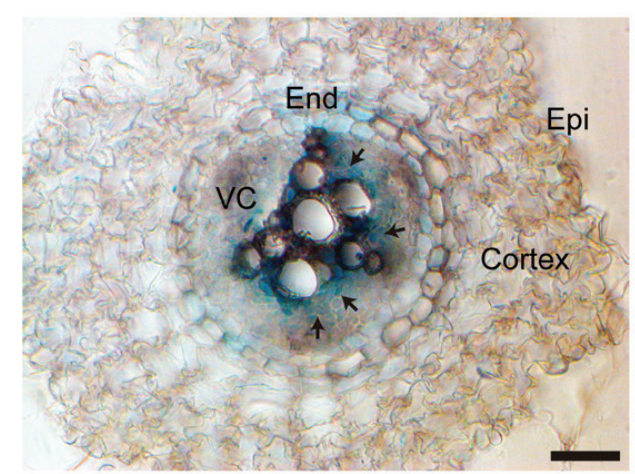

Fig. 1. MtYSL3 is highly expressed in nodule and root vasculature. (A) Phylogenetic tree of the Group I YSL transporters, MtYSL1-MtYSL4 (Medtr1g077840, Medtr1g007540, Medtr3g092090, and Medtr1g007580, respectively) and their representative homologues in Zea mays (ZmYS1, Zm00001d017429; and ZmYSL2, Zm00001d025977), Oryza sativa (OsYSL15, Os02g0650300; and OsYSL16, Os04g0542800), Brachypodium distachyon (BdYS1A, BRADI_3g50267; BdYS1B, BRADI_3g50263; BdYSL2, BRADI_3g50260; and BdYSL3, BRADI_5g17230), and Arabidopsis thaliana (AtYSL1, At4g24120; AtYSL2, At5g24380; and AtYSL3, At5g53550). (B) Gene expression relative to the internal standard gene ubiquitin carboxyl-terminal hydrolase in 28 dpi shoots, roots, and nodules of nitrogen-fertilized plants and nodulated plants. Data are the mean \pm SE of five independent experiments. (C) Histochemical staining of GUS activity in 28 dpi roots and nodules of $M$. truncatula plants transformed with a vector containing the MtYSL3-promoter::gus. Scale bar=500 $\mu \mathrm{m}$. (D) Longitudinal section of a GUS-stained 28 dpi nodule expressing gus under the MtYSL3 promoter. Scale bar=200 $\mu \mathrm{m}$. (E) Cross-section of a GUS-stained nodule expressing gus under the MtYSL3 promoter. Scale bar=200 $\mu \mathrm{m}$. (F) Crosssection of a GUS-stained root expressing gus under the MtYSL3 promoter. End, endodermis; Epi, epidermis; VC, vascular cylinder. Scale bar=50 $\mu$ m.

\section{MtYSL3 is involved in symbiotic nitrogen fixation}

To determine the role of MtYSL3 in M. truncatula physiology, two Tnt 1 insertion lines were obtained from the Noble
Research Institute (Tadege et al., 2008). NF17945 ( $y$ sl3-1) has an insertion in position +342 , within the first exon of the gene (Fig. 3A). NF12068 ( $y$ sl3-2) is inserted in the promoter region 
A

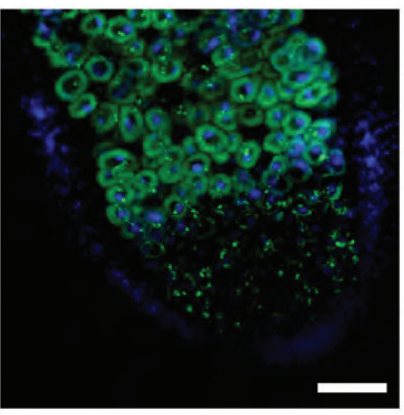

B

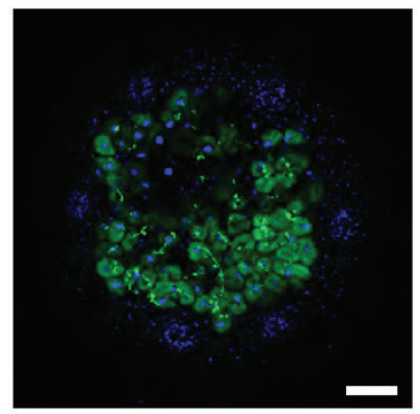

C

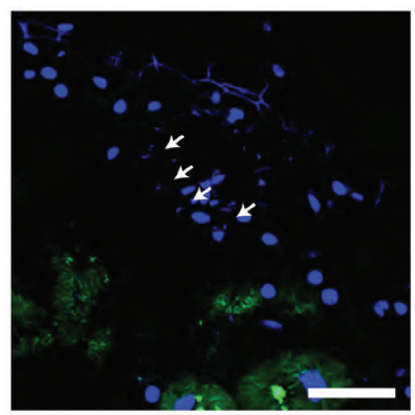

D

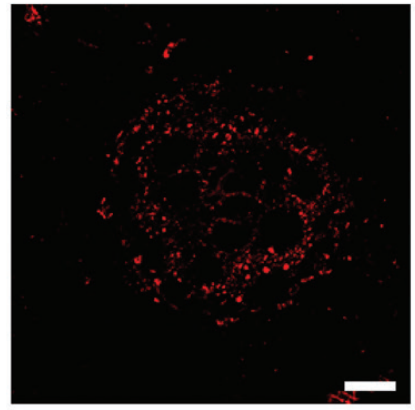

E

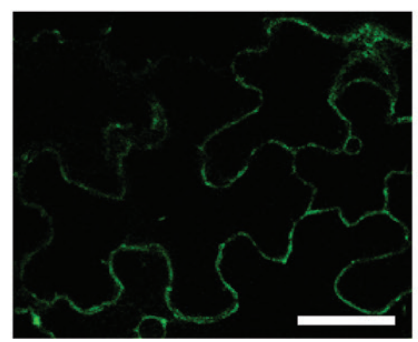

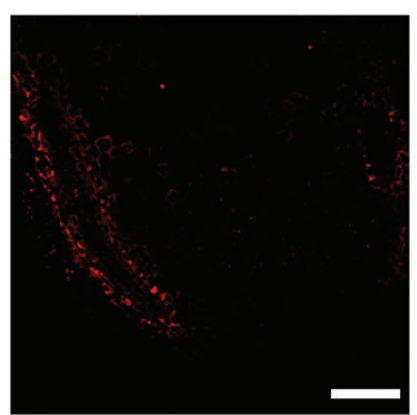
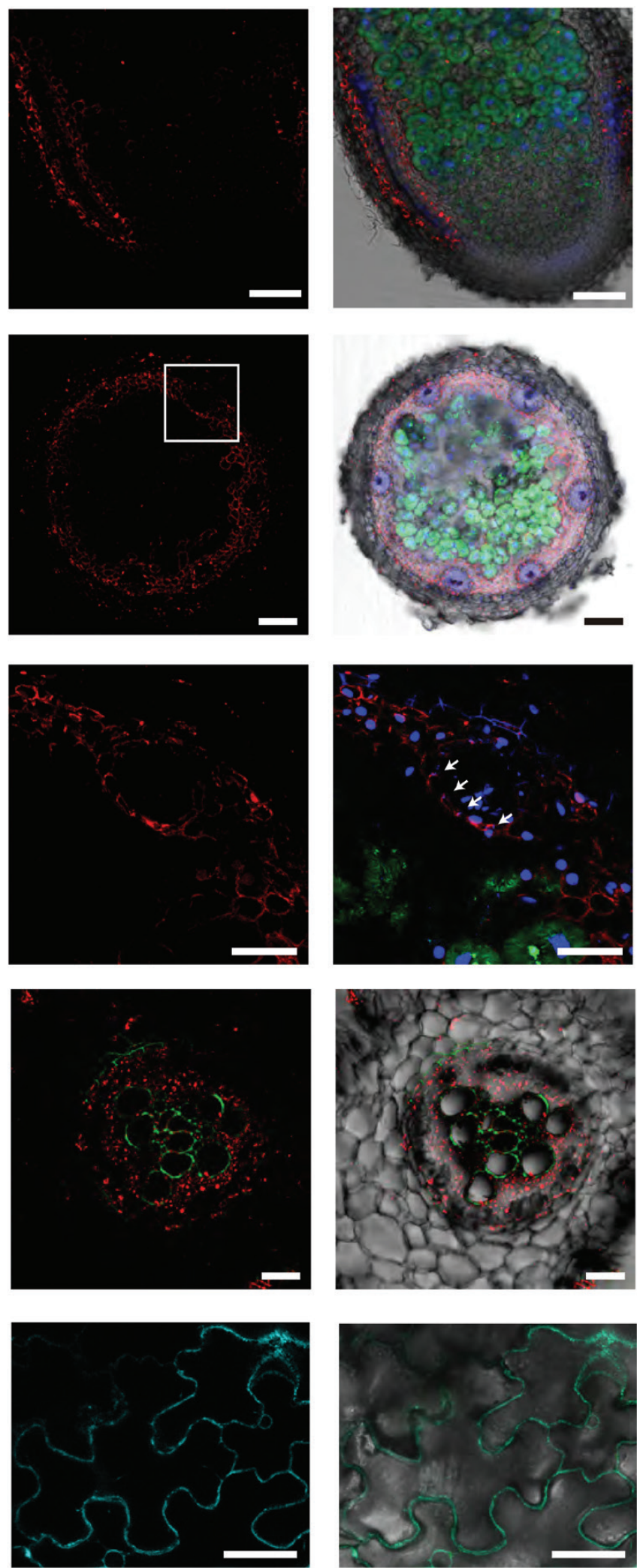
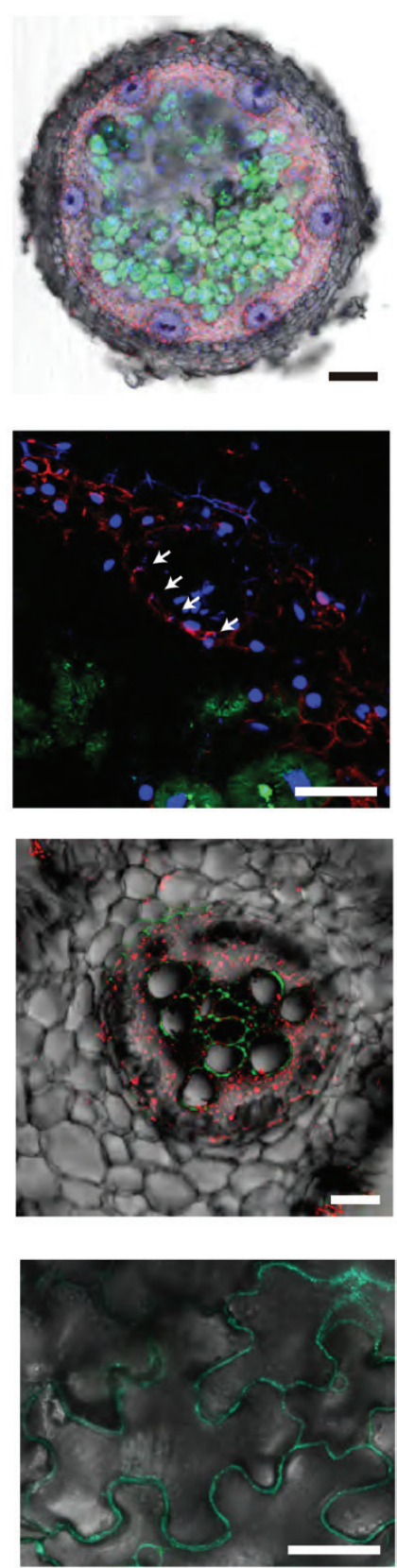

Fig. 2. MtYSL3-HA is located in the plasma membrane of endodermal cells in roots and nodules, in root xylem parenchyma, and in the nodule cortical cells. (A) Longitudinal section and (B) cross-section of $28 \mathrm{dpi} M$. truncatula nodules colonized with S. meliloti constitutively expressing GFP (green) and transformed with a vector expressing the fusion MtYSL3-HA under the regulation of its endogenous promoter. MtYSL3-HA localization was determined using an Alexa 594-conjugated antibody (red). DNA was stained with DAPI (blue). Left panels show the overlay of GFP and DAPI channels; centre panels the Alexa594 channel; and right panels the overlay of GFP, Alexa594, and DAPI channels with the brightfield image. Scale bars=200 $\mu \mathrm{m}(\mathrm{A})$ or $50 \mu \mathrm{m}(\mathrm{B})$. (C) Magnification of the vascular bundle within the boxed region indicated in (B). Left panel shows the overlay of GFP and DAPI channels; centre panel the Alexa594 channel; and right panel the overlay of the three channels. Arrows indicate the position of the autofluorescence signal of the Casparian strip. Scale bars $=50 \mu \mathrm{m}$. (D) Cross-section of an M. truncatula root transformed with a vector expressing the fusion MtYSL3-HA under regulation of its 
of MtYSL3, in position -19. While in both cases MtYSL3 expression was detected, these Tnt 1 lines showed a severe reduction of MtYSL3 transcript compared with wild-type plants (Fig. 3B). Transposon insertion in $y s l 3-1$ resulted in an altered splicing that left a 30 nucleotide insertion of the Tnt1 sequence in MtYSL3 mRNA. As a result, five amino acids were mutated (Y115D, S116D, I117V, A118H, and G120L) and four more were added between amino acids 118 and 119 (LIEE). These changes occurred in the third predicted transmembrane domain, and would probaby disrupt this region, as indicated by the transmembrane region prediction software Phobius (http://phobius.sbc.su.se/) (Supplementary Fig. S7). As a result of this change, two transmembrane helices ( 2 and 3 ) would be lost. This type of change would cause a major disruption on the functionality of any membrane protein, and thus $y s l 3-1$ has been considered as a loss-of-function mutant, while $y$ sl3-2 would be a knockdown line.

Under non-symbiotic conditions, when the plants were not inoculated with rhizobia but fertilized with ammonium nitrate, no significant differences were observed in plant growth and biomass production between wild-type plants, Tnt 1 segregants with two wild-type copies of MtYSL3 (+/+ lines), or segregants with both MtYSL3 copies mutated (-/- lines) (Fig. 3C, D). No significant differences were observed in either total chlorophyll content (Fig. 3E) or iron concentration in shoots (Fig. 3F). However, $y s l 3$ plants had a trend to accumulate more iron in roots, significatively so in the $y s l 3-2$ allele. Copper levels were not significantly different in shoots or roots (Fig. 3G), while zinc concentrations were significantly higher in the roots of both MtYSL3 mutants (Fig. 3H). Unlike A. thaliana orthologues (Waters et al., 2006), MtYSL3 mutant plants did not show any significant reduction in fertility, as indicated by the number of pods per plant and of seeds within them (Supplementary Fig. S8).

However, when the nitrogen was provided by the endosymbiotic rhizobia within their root nodules, $y$ sl3-1 and $y s l 3-2$ -/- lines had a reduced growth when compared with their wild-type segregants (Fig. 4A). This was also shown when comparing the dry weight of loss-of-function $y$ sl3-1-/- with the wild-type segregant, with significantly lower biomass production (Fig. 4B). While there were no significant changes in the number of nodules per plant (Fig. 4C), both mutant lines had $\sim 60 \%$ of the nitrogenase activity of the wild-type control or $+/+$ segregant lines (Fig. 4D). This could be due to the reduced iron content in nodules of $y$ sl3-1 and $y$ sl32 plants (Fig. 4E). While the copper concentration did not change significantly in these organs, ysl3-2 nodules had less zinc (Fig. 4F, G). However, copper was more concentrated in $y s l 3-1$ shoots. Similarly to non-inoculated plants, there were no significant difference in pod or seed production in these plants compared with their controls (Supplementary Fig. S9). In mutant $y s l 3-1$ nodules, no changes in the expression of
MtYSL2 were observed, while MtYSL1 was down-regulated (Supplementary Fig. S10). Supplementation of the nutrient solution with higher zinc or iron levels did not improve the nitrogenase activity in mutant nodules (Supplementary Fig. S11). Nodule development did not seem to be substantially affected by the MtYSL3 mutations in terms of either nodulation kinetics (Supplementary Fig. S12) or the overall nodule anatomy (Supplementary Fig. S13).

\section{Mutation of MtYSL3 affects iron and zinc distribution}

The reduced iron content in $y s / 3$ nodules and lower nitrogenase activity could be the result of less iron being delivered to the fixation zone. To test this possibility, we carried out X-ray fluorescence tests in nodules from wild-type and $y$ sl3-1 nodules, using calcium to delimit the nodule walls and to identify the main developmental zones (Fig. 5A). While a typical wild-type nodule has less iron in the apical region relative to the fixation zone (Fig. 5B), ysl3-1 nodules had the opposite, indicating that not enough iron would be reaching the fixation zone. Furthermore, while significant changes in nodule zinc concentration were observed only in $y s l 3-2$ nodules, zinc distribution was also affected in $y s l 3-1$ nodules. X-ray fluorescence data showed that this nutrient accumulated at much higher levels in the $y s l 3-1$ nodule vessels than in the wild type (Fig. 5A, C). Although metal-NA would be the likely substrate of MtYSL3, no significant differences in NA concentration in ysl3-1 nodules were observed when compared with the wild type (Supplementary Fig. S14).

\section{Discussion}

Transition metals are essential plant nutrients (Marschner, 2011). Typically, the main plant metal sinks are in the leaves, where these elements participate in the electron transport chains in photosynthesis and oxidative respiration, among several other processes, and in the seeds, where they are critical for embryo development and germination (Kobayashi and Nishizawa, 2012; Yruela, 2013; Ibeas et al., 2017). Consequently, plants dedicate a large effort to ensure metal translocation from roots to leaves and seeds, which includes the participation of Arabidopsis YSL1 and YSL3 (Curie et al., 2009; Conte et al., 2013). However, this is more challenging in legumes when they are in symbiosis with rhizobia. Due to the large amounts of metalloenzymes participating in symbiotic nitrogen fixation (Brear et al., 2013; González-Guerrero et al., 2014), nodules are a major metal sink. A third of the total plant iron content and a quarter of the total copper and zinc are concentrated in nodules, which, in the case of M. truncatula, represents $\sim 5 \%$ of the total plant biomass (Tejada-Jiménez et al., 2015; Abreu et al., 2017; Senovilla et al., 2018). Therefore,

endogenous promoter located with an Alexa594-conjugated antibody (red, left panel). The centre panel shows it co-localization with the autofluorescence signal of lignin (green). The right panel shows the overlaid images with the transillumination channel. Scale bars=50 $\mu \mathrm{m}$. (E) Transient expression of MtYSL3-GFP and AtPIP2-CFP in N. benthamiana leaves. The left panel shows the localization of MtYSL3 fused to GFP (green) in tobacco cells. The middle panel shows the localization of the plasma membrane marker AtPIP2 fused to CFP in the same cells. The right panel is the overlay of the two previous channels together with the bright field image. Scale bars $=50 \mu \mathrm{m}$. 
A $y s / 3-1$

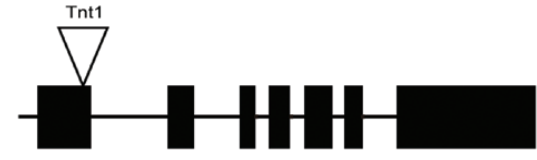

ys/3-2

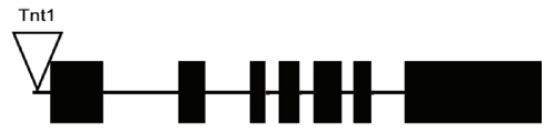

C

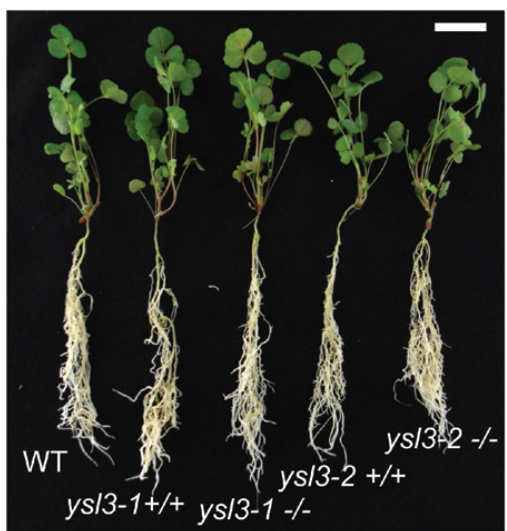

E

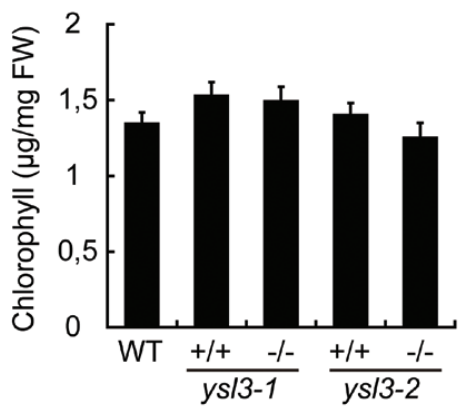

G

-WT ys/3-1 ys/3-2

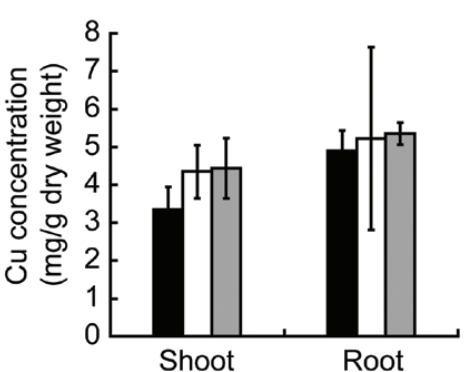

B
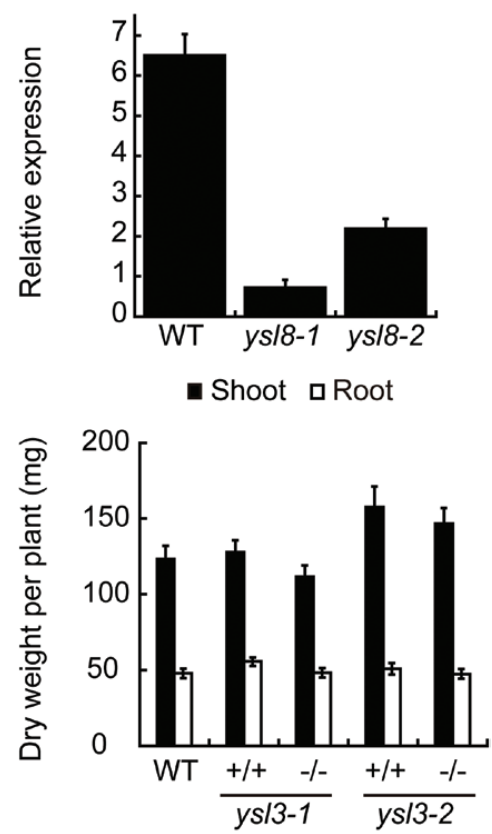

F

-WT ys/3-1 ys/3-2

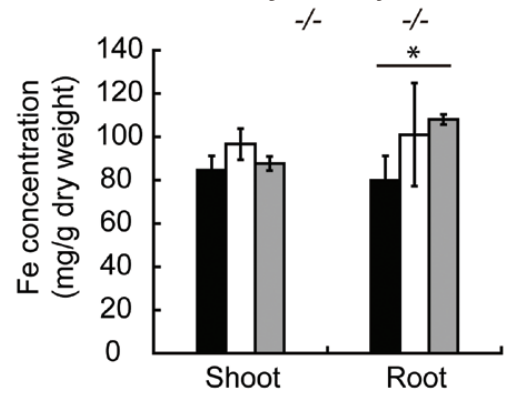

H

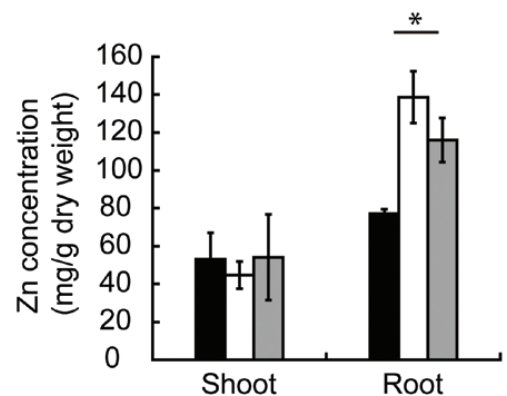

Fig. 3. MtYSL3 does not play an important role under non-symbiotic conditions. (A) Position of the Tnt1 insertion site for ys/3-1 (NF17945) and for ys/3-2 (NF12068). (B) RT-PCR of MtYSL3 expression in 28 dpi nodules in wild-type (WT), ys/3-1, and ys/3-2 M. truncatula lines. Expression of ubiquitin carboxylterminal hydrolase was used as the positive control. (C) Growth of representative WT, ys/3-1, and ys/3-2 plants. +/+ indicates ys/3 segregants with two WT copies of MtYSL3, while -/- indicate that both copies have the Tnt1 insertion. Scale bar=3 cm. (D) Dry weight of shoots and roots of 28 dpi plants. Data are the mean \pm SE ( $n=20-60$ plants). (E) Chlorophyll content of WT, ys/3-1 +/+, ys/3-1 -/-, ys/3-2 +/+, and ys/3-2 -/-. Data are the mean \pm SE of 10 sets of 4-5 pooled plants. (F) Iron content in roots and shoots of WT, ys/3-1 -/-, and ys/3-2 -/- plants. Data are the mean \pm SE of three pools of the indicated organs collected from 4-5 plants. (G) Copper content in roots and shoots of WT, ys/3-1 -/-, and ys/3-2 -/- plants. Data are the mean \pm SE of three pools of the indicated organs collected from 4-5 plants. $(H)$ Zinc content in roots and shoots of WT, ys/3-1 -/-, and ys/3-2 -/- plants. Data are the mean \pm SE of three pools of the indicated organs collected from 4-5 plants. * indicates statistically significant differences $(P<0.05)$. 
legumes have to direct large quantities of metals not only to shoots, but also to nitrogen-fixing nodules. In this task, it is likely that YSL proteins similar to $A$. thaliana YSL1 and YSL3 would participate.

MtYSL3 is one of the four clade I YSLs in M. truncatula. It is closely related to $A$. thaliana YSL1 and YSL3, both being responsible for long-distance metal delivery (Walker and Waters, 2011) (Fig. 1A). As was the case for the A. thaliana orthologues (Waters et al., 2006), MtYSL3 is expressed in the vasculature, both in roots and in nodules (Fig 1C-F). This was confirmed by immunolocalization of a HA-tagged protein using confocal microscopy (Fig. 2A-D). Moreover, MtYSL3-HA had a plasma membrane localization in endodermal and in root xylem parenchyma cells (Fig 2B-E). In nodules, some expression could also be detected in nodule cortical cells, those in the exterior of the nodule (Fig. 2B). This localization would be consistent with a role in vascular transition metal transport, as well as metal uptake by nodule cortical cells.

In non-inoculated plants, MtYSL3 does not seem to play a critical role by itself (Fig. 3). There are no major changes in biomass production, leaf chlorosis, or plant fertility. There is a significant accumulation in roots of zinc in both mutant alleles and of iron in one of them (Fig. 3F, H). However, this had no effect on total shoot metal concentrations. This is consistent with the reported functional redundancy of the YSL family. It could be hypothesized that closely related MtYSL2 could carry out this function since it is expressed in roots and nodules (Supplementary Fig. S1), and in A. thaliana, AtYSL1 (orthologue to MtYSL1) is able to partially compensate for the loss of AtYSL3 (Chu et al., 2010). However, when M. truncatula is nodulated, losing or simply reducing MtYSL3 expression results in a $40 \%$ reduction of nitrogenase activity, with a significant decrease of biomass production when MtYSL3 is inactivated (Fig. 4A-D). Moreover, iron and zinc accumulation and distribution are affected in the MtYSL3 mutants (Figs 4E, $\mathrm{G}, 5)$. Less iron reaches $y s l 3-1$ and $y s l 3-2$ nodules, and it is less abundant in the fixation zone which would result in less iron being available for nitrogenase cofactor synthesis. Zinc is retained in the nodule vessels (Fig. 5B), which would result in lower amounts available for nodule functioning, although the precise role of zinc in nodule functioning has not yet been determined.

Therefore, it could be proposed that MtYSL3 would be participating in iron and zinc delivery to nodules, considering that iron levels were reduced in nodules, and zinc became trapped in the veins of $y s 13$ (Figs 4E, 5). However, MtYSL3 is probably not the only transporter mediating this process. Any major disruption of iron, copper, or zinc delivery to nodules results in a more severe reduction of nitrogenase activity, the presence of white, non-functional nodules, and/or a reduction in their size (Tejada-Jiménez et al., 2015, 2017; Abreu et al., 2017; Kryvoruchko et al., 2018; Senovilla et al., 2018). In contrast, the $y s l 3$ phenotype was milder (Fig. 4; Supplementary Figs S12, S13). This would indicate that another transporter might be involved in metal delivery from the vasculature. Based on the available transcriptomic data, MtYSL2 (highly expressed in nodules, but five times less so than MtYSL3) could be participating (Supplementary Fig. S1). However, its expression was not increased to compensate for MtYSL3 mutation (Supplementary Fig. S10). This would indicate that MtYSL2 has different transcriptional control in nodules compared with MtYSL3, which might also reflect a different functional role.

The localization of MtYSL3 (Figs 1,2) also indicates additional roles to delivering metals to nitrogen-fixing nodule cells. If this were its unique function, MtYSL3 would only be expressed in the vascular region in the infection/differentiation zone of the nodule, since this is the region where plant-delivered transition metals are released (Rodríguez-Haas et al., 2013). In contrast, as shown for the molybdate vascular transporter MtMOT1.2 (Gil-Díez et al., 2019), MtYSL3 is located along the whole nodule vessels, including the fixation zone. Moreover, no polar distribution of the transporter was observed (Fig. 2), which suggests that mass effect would drive the net direction of the MtYSL3 substrate, as proposed for MtMOT1.2. This would be compatible with MtYSL3 being involved in recovering iron and zinc from the apoplast in Zone III, a role of particular importance when considering the prevalent low metal bioavailability in soils (Kim and Guerinot, 2007; Alloway, 2008). However, this metal recovery capability would not be essential either, since none of the MtYSL3 mutants had any alteration in fertility (Supplementary Figs S8, S9), as was reported when both AtYSL1 and AtYSL3 were mutated (Waters et al., 2006). This is in contrast to the proposed role of NA in metal recycling from senescent nodules (Hakoyama et al., 2009). All this evidence also indicates the existence of a redundant protein that would be carrying out this function together with MtYSL3.

In addition to the vasculature, MtYSL3 is also located in the cortical nodule cells, although expressed at lower levels (Fig 2B). There, it could be introducing metal-NA complexes (including iron-NA and zinc-NA) into these cells. For iron, this is in contrast to rhizobia-infected cells, which introduce iron as $\mathrm{Fe}^{2+}$ through an Nramp protein (TejadaJiménez et al., 2015), with citrate playing an important role in its solubility in the apoplast (Takanashi et al., 2013; Kryvoruchko et al., 2018). This would indicate the existence of different iron pools to separate a limiting nutrient with different physiological functions. Previous data on zinc transporter MtZIP6, only expressed in rhizobia-infected cells, also hints at at least a partial tissue specialization of metal transport (Abreu et al., 2017). However, work on in situ metal speciation analyses should shed some light on this possibility once synchrotron-based X-ray absorption nearedge structure (XANES) reaches the required sensitivity and resolution and opens up the study of the mechanisms of intertissue metal sorting in nodules.

In summary, our data indicate that MtYSL3 is involved in vascular transition metal delivery, probably iron and zinc, to nitrogen-fixing nodule cells, as well as uptake by cortical cells. Our data also suggest that at least an additional metal transporter is performing analogous functions, since the $y$ sl3 phenotype is relatively mild. Future work will be directed towards unveiling these additional transporters. 
A

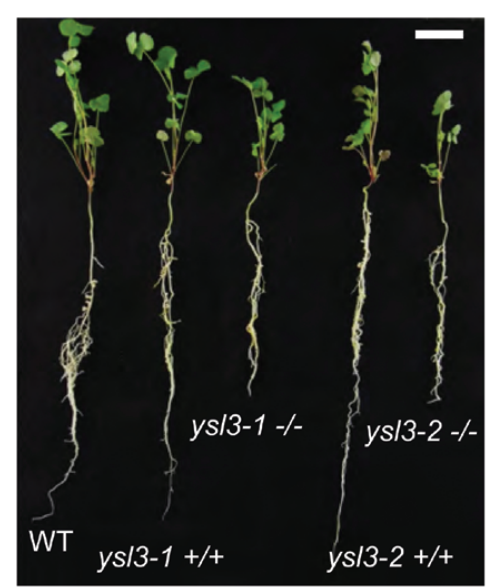

C

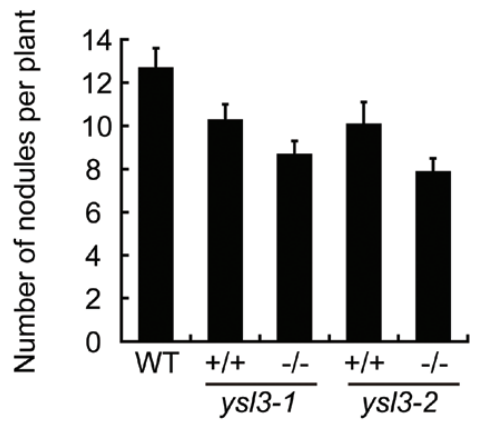

E

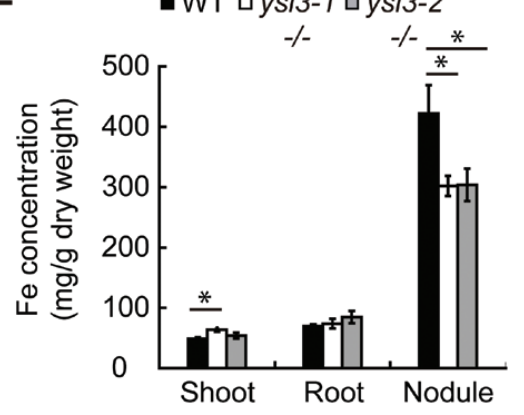

G

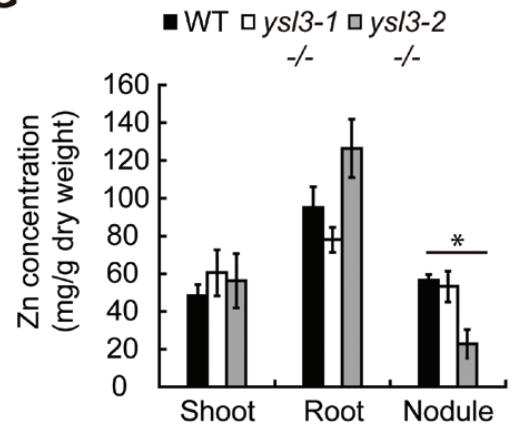

B

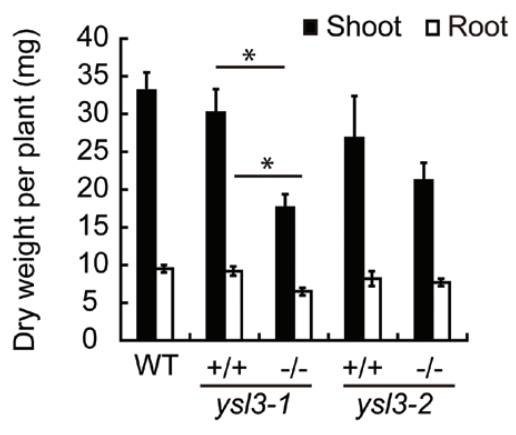

D

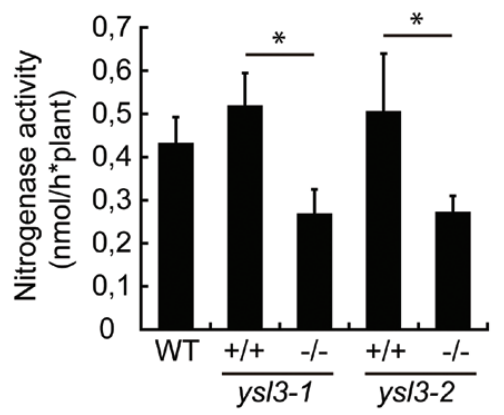

F

-WT ys/3-1 ys/3-2

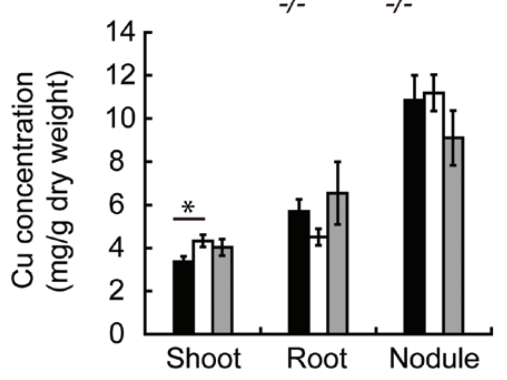

Fig. 4. MtYSL3 participates in symbiotic nitrogen fixation. (A) Representative wild-type (WT), ys/3-1, and ys/3-2 plants. +/+ indicates ys/3 segregants with two WT copies of MtYSL3, while -/- indicate that both copies have the Tnt1 insertion. Scale bar=3 cm. (B) Dry weight of shoots and roots of 28 dpi plants. Data are the mean $\pm S E(n=10-40$ plants). (C) Number of nodules per plant in WT and mutant lines. Data are the $\pm S E(n=10-40$ plants). (D) Nitrogenase activity in 28 dpi nodules from WT, ys/3-1, and ys/3-2 plants. Data are the mean $\pm S E(n=5-15$ sets of pooled plants). (E) Iron content in shoots, roots, and nodules of WT, ys/3-1 -/-, and ys/3-2 -/- plants. Data are the mean \pm SE of three pools of the indicated organs collected from 4-5 plants. (F) Copper content in shoots, roots, and nodules of WT, ys/3-1 -/-, and ys/3-2 -/- plants. Data are the mean \pm SE of three pools of the indicated organs collected from 4-5 plants. (G) Zinc content in roots and shoots of WT, ys/3-1-/-, and ys/3-2 -/- plants. Data are the mean \pm SE of three pools of the indicated organs collected from $4-5$ plants. ${ }^{*}$ indicates statistically significant differences $(P<0.05)$. 
A
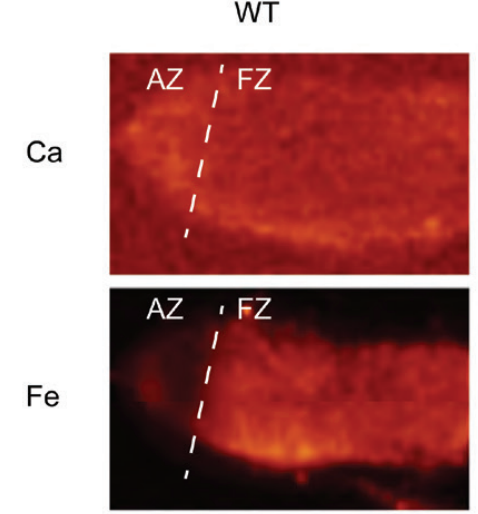

$\mathrm{Zn}$

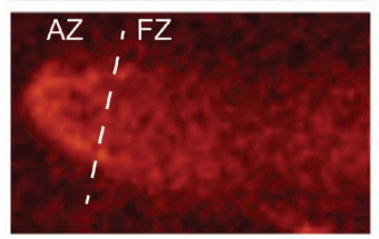

B

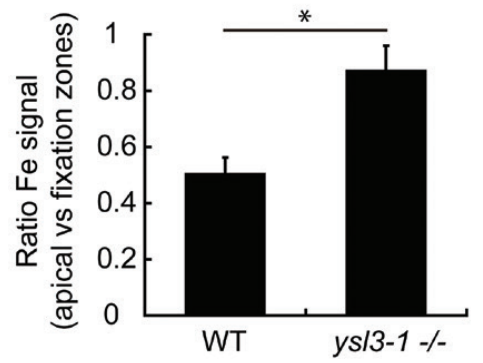

$y s / 3-1-$

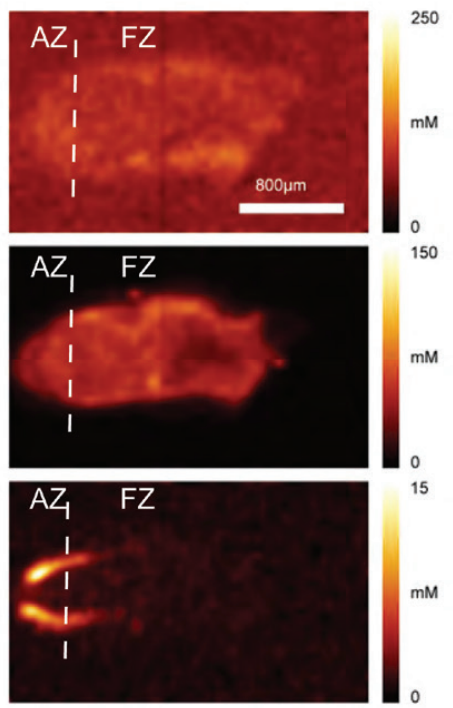

C

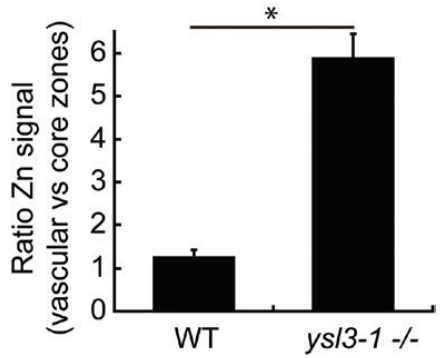

Fig. 5. Mutation of MtYSL3 alters nodule iron and zinc distribution. (A) X-ray fluorescence localization of calcium (top panels), iron (centre panels), and zinc (lower panels) in representative 28 dpi nodules from wild-type (WT) and ys/3-1 -/- plants. AZ indicates the apical zone, and FZ the fixation zone of the nodules. (B) Ratio of iron concentration in the apical versus the fixation zone in 28 dpi nodules from WT and ys/3-1 -/- plants. Data are the mean $\pm S E$ ( $n=4-5$ nodules). (C) Ratio of zinc concentration in the nodule core versus the vasculature in 28 dpi nodules from WT and ys/3-1 -/- plants. Data are the mean \pm SE $\left(n=4-5\right.$ nodules). ${ }^{*}$ indicates statistically significant differences $(P<0.05)$.

\section{Supplementary data}

The following supplementary data are available at JXB online.

Fig. S1. Group I M. truncatula YSL expression.

Fig. S2. Expression of MtYSL3 during nodule development.

Fig. S3. Histochemical staining of GUS activity in 28 dpi roots of M. truncatula transformed with MtYSL3promoter::gus.

Fig. S4. MtYSL3-HA is located in the plasma membrane of endodermal cells in the nodule vasculature.

Fig. S5. Autofluorescence control for Alexa594 signal.

Fig. S6. Control for antibody specificity

Fig. S7. Effect of $y$ sl3-1 Tnt 1 insertion in MtYSL3 topology.

Fig. S8. Effect of MtYSL3 mutation in plant fertility under non-symbiotic conditions.

Fig. S9. Effect of MtYSL3 mutation in plant fertility under symbiotic conditions.

Fig. S10. Expression of MtYSL1 and MtYSL2 in $\gamma s 13-1$ nodules.

Fig. S11. Metal fortification does not rescue $y s l 3$ mutants.

Fig. S12. Time-course nodulation assay.

Fig. S13. Sections of 28 dpi wild-type, $y s l 3-1$, and $y s l 3-2$ nodules.

Fig. S14. Nicotianamine content in 28 dpi wild-type, $y s l 3-1$, and $y$ sl3-2 nodules.

Table S1. Primers used in this study.

\section{Data availability}

All data supporting the findings of this study are available within the paper and within its supplementary data published online.

\section{Acknowledgements}

This research was funded by a European Research Council Starting Grant (ERC-2013-StG-335284) and the Spanish State Research Agency (AEI) grant (AGL2015-65866-P) to MGG, and a AEI grant (AGL2016-75226-R) to JA and AA-F co-financed by the European Regional Development Fund (FEDER). RC-R and FJJ-P were supported by a Formación del Personal Investigador fellowship (BES2013-062674 and BES-2017-082913, respectively). IA was the recipient of a Juan de la Cierva- Formación postdoctoral fellowship from Ministerio de Ciencia, Innovación y Universidades (FJCI-201733222). VE was partially funded by the Severo Ochoa Programme for Centres of Excellence in R\&D from the AEI (grant SEV-20160672) received by the Centro de Biotecnología y Genómica de Plantas (UPM-INIA). Development of the M. truncatula Tnt 1 mutant population was, in part, funded by the National Science Foundation, USA (DBI-0703285) to KSM. AM and HK and the $\mu$ XRF measurements were supported by the Ministry of Education, Youth and Sports of the Czech Republic with co-financing from the European Union (grant 'KOROLID', CZ.02.1.01/0.0/0.0/15_003/0000336) and the 
Czech Academy of Sciences (RVO: 60077344). We would also like to acknowledge the other members of laboratory 281 at Centro de Biotecnología y Genómica de Plantas (UPM-INIA) for their support and feedback in preparing this manuscript.

\section{Author contributions}

RC-R and IA carried out most of the experimental work. MR,AM, and HK also contributed to XRF assays. LN-A performed the atomic absorption spectroscopy determinations. VE assisted in the nodulation kinetic assays and helped in the expression assays. FJJ-P, JA, and AA-F contributed to the NA determinations. JW and KSM identified the YSL3 mutants. RC-R, IA, JI, and MG-G designed and analysed the assays. MG-G directed the research and wrote the manuscript with input from all authors.

\section{References}

Abreu I, Saez A, Castro-Rodríguez R, et al. 2017. Medicago truncatula Zinc-Iron Permease6 provides zinc to rhizobia-infected nodule cells. Plant, Cell \& Environment 40, 2706-2719.

Alloway BJ. 2008. Zinc in soils and crop nutrition, 2nd edn. International Zinc Association and International Fertilizer Industry Association.

Andrés-Colás N, Sancenón V, Rodríguez-Navarro $\mathrm{S}$, Mayo $\mathrm{S}$, Thiele DJ, Ecker JR, Puig S, Peñarrubia L. 2006. The Arabidopsis heavy metal P-type ATPase HMA5 interacts with metallochaperones and functions in copper detoxification of roots. The Plant Journal 45, 225-236.

Appleby CA. 1984. Leghemoglobin and Rhizobium respiration. Annual Review of Plant Physiology 35, 443-478.

Avenhaus U, Cabeza RA, Liese R, Lingner A, Dittert K, SalinasRiester G, Pommerenke C, Schulze J. 2016. Short-term molecular acclimation processes of legume nodules to increased external oxygen concentration. Frontiers in Plant Science 6, 1012.

Banakar R, Alvarez Fernández Á, Abadía J, Capell T, Christou P. 2017. The expression of heterologous Fe (III) phytosiderophore transporter HVYS1 in rice increases Fe uptake, translocation and seed loading and excludes heavy metals by selective Fe transport. Plant Biotechnology Journal 15, 423-432.

Boisson-Dernier A, Chabaud M, Garcia F, Bécard G, Rosenberg C, Barker DG. 2001. Agrobacterium rhizogenes-transformed roots of Medicago truncatula for the study of nitrogen-fixing and endomycorrhizal symbiotic associations. Molecular Plant-Microbe Interactions 14, 695-700.

Brear EM, Bedon F, Gavrin A, Kryvoruchko IS, Torres-Jerez I, Udvardi MK, Smith PMC. 2020. GmVTL1a is an iron transporter on the symbiosome membrane of soybean with an important role in nitrogen fixation. New Phytologist. doi: 10.1111/nph.16734

Brear EM, Day DA, Smith PM. 2013. Iron: an essential micronutrient for the legume-rhizobium symbiosis. Frontiers in Plant Science 4, 359.

Brito B, Palacios JM, Hidalgo E, Imperial J, Ruiz-Argüeso T. 1994. Nickel availability to pea (Pisum sativum L.) plants limits hydrogenase activity of Rhizobium leguminosarum bv. viciae bacteroids by affecting the processing of the hydrogenase structural subunits. Journal of Bacteriology 176, 5297-5303.

Cheng HP, Walker GC. 1998. Succinoglycan is required for initiation and elongation of infection threads during nodulation of alfalfa by Rhizobium meliloti. Journal of Bacteriology 180, 5183-5191.

Chu HH, Chiecko J, Punshon T, Lanzirotti A, Lahner B, Salt DE, Walker EL. 2010. Successful reproduction requires the function of Arabidopsis Yellow Stripe-Like1 and Yellow Stripe-Like3 metalnicotianamine transporters in both vegetative and reproductive structures. Plant Physiology 154, 197-210.

Conte SS, Chu HH, Rodriguez DC, Punshon T, Vasques KA, Salt DE, Walker EL. 2013. Arabidopsis thaliana Yellow Stripe1-Like4 and Yellow Stripe1-Like6 localize to internal cellular membranes and are involved in metal ion homeostasis. Frontiers in Plant Science 4, 283.

Conte SS, Walker EL. 2011. Transporters contributing to iron trafficking in plants. Molecular Plant 4, 464-476.
Curie C, Cassin G, Couch D, Divol F, Higuchi K, Le Jean M, Misson J, Schikora A, Czernic P, Mari S. 2009. Metal movement within the plant: contribution of nicotianamine and yellow stripe 1-like transporters. Annals of Botany 103, 1-11.

Curie C, Panaviene Z, Loulergue C, Dellaporta SL, Briat JF, Walker EL. 2001. Maize yellow stripe1 encodes a membrane protein directly involved in $\mathrm{Fe}(\mathrm{III})$ uptake. Nature 409, 346-349.

Deblaere R, Bytebier B, De Greve H, Deboeck F, Schell J, Van Montagu M, Leemans J. 1985. Efficient octopine Ti plasmid-derived vectors for Agrobacterium-mediated gene transfer to plants. Nucleic Acids Research 13, 4777-4788.

Downie JA. 2014. Legume nodulation. Current Biology 24, R184-R190.

Escudero V, Abreu I, Del Sastre E, et al. 2020a. Nicotianamine synthase 2 is required for symbiotic nitrogen fixation in Medicago truncatula nodules. Frontiers in Plant Science 10, 1780.

Escudero V, Abreu I, Tejada-Jiménez M, et al. 2020b. Medicago truncatula Ferroportin2 mediates iron import into nodule symbiosomes. New Phytologist 228, 194-209.

Flis P, Ouerdane L, Grillet L, Curie C, Mari S, Lobinski R. 2016. Inventory of metal complexes circulating in plant fluids: a reliable method based on HPLC coupled with dual elemental and high-resolution molecular mass spectrometric detection. New Phytologist 211, 1129-1141.

Gil-Díez P, Tejada-Jiménez M, León-Mediavilla J, Wen J, Mysore KS, Imperial J, González-Guerrero M. 2019. MtMOT1.2 is responsible for molybdate supply to Medicago truncatula nodules. Plant, Cell \& Environment 42, 310-320.

González-Guerrero M, Escudero V, Sáez Á, Tejada-Jiménez M. 2016. Transition metal transport in plants and associated endosymbionts. Arbuscular mycorrhizal fungi and rhizobia. Frontiers in Plant Science $\mathbf{7}$, 1088.

González-Guerrero M, Matthiadis A, Sáez Á, Long TA. 2014. Fixating on metals: new insights into the role of metals in nodulation and symbiotic nitrogen fixation. Frontiers in Plant Science 5, 45.

Hakoyama T, Watanabe $\mathrm{H}$, Tomita J, Yamamoto A, Sato S, Mori $\mathrm{Y}$, Kouchi H, Suganuma N. 2009. Nicotianamine synthase specifically expressed in root nodules of Lotus japonicus. Planta 230, 309-317.

Hardy RW, Holsten RD, Jackson EK, Burns RC. 1968. The acetyleneethylene assay for $\mathrm{N}_{2}$ fixation: laboratory and field evaluation. Plant Physiology 43, 1185-1207.

Hussain D, Haydon MJ, Wang Y, Wong E, Sherson SM, Young J, Camakaris J, Harper JF, Cobbett CS. 2004. P-type ATPase heavy metal transporters with roles in essential zinc homeostasis in Arabidopsis. The Plant Cell 16, 1327-1339.

Ibeas MA, Grant-Grant S, Navarro N, Perez MF, Roschzttardtz H. 2017. Dynamic subcellular localization of iron during embryo development in Brassicaceae seeds. Frontiers in Plant Science 8, 2186.

Jurkevitch E, Hadar Y, Chen Y. 1992. Differential siderophore utilization and iron uptake by soil and rhizosphere bacteria. Applied and Environmental Microbiology 58, 119-124.

Kakar K, Wandrey M, Czechowski T, et al. 2008. A community resource for high-throughput quantitative RT-PCR analysis of transcription factor gene expression in Medicago truncatula. Plant Methods 4, 18.

Kereszt A, Mergaert P, Kondorosi E. 2011. Bacteroid development in legume nodules: evolution of mutual benefit or of sacrificial victims? Molecular Plant-Microbe Interactions 24, 1300-1309.

Kim SA, Guerinot ML. 2007. Mining iron: iron uptake and transport in plants. FEBS Letters 581, 2273-2280.

Kobayashi T, Nishizawa NK. 2012. Iron uptake, translocation, and regulation in higher plants. Annual Review of Plant Biology 63, 131-152.

Kryvoruchko IS, Routray P, Sinharoy S, et al. 2018. An iron-activated citrate transporter, MtMATE67, is required for symbiotic nitrogen fixation. Plant Physiology 176, 2315-2329.

Kumar RK, Chu HH, Abundis C, Vasques K, Rodriguez DC, Chia JC, Huang R, Vatamaniuk OK, Walker EL. 2017. Iron-nicotianamine transporters are required for proper long distance iron signaling. Plant Physiology 175, 1254-1268.

Kumar S, Stecher G, Tamura K. 2016. MEGA7: molecular evolutionary genetics analysis version 7.0 for bigger datasets. Molecular Biology and Evolution 33, 1870-1874. 
Lubkowitz M. 2011. The oligopeptide transporters: a small gene family with a diverse group of substrates and functions? Molecular Plant 4, 407-415.

Marschner P. 2011. Mineral nutrition of higher plants, 3rd edn. Academic Press. Amsterdam.

Mijovilovich A, Morina F, Bokhari SN, Wolff T, Küpper H. 2020. Analysis of trace metal distribution in plants with lab-based microscopic $\mathrm{X}$-ray fluorescence imaging. Plant Methods 16, 82.

Morrissey J, Baxter IR, Lee J, Li L, Lahner B, Grotz N, Kaplan J, Salt DE, Guerinot ML. 2009. The ferroportin metal efflux proteins function in iron and cobalt homeostasis in Arabidopsis. The Plant Cell 21, 3326-3338.

Nakagawa T, Kurose T, Hino T, Tanaka K, Kawamukai M, Niwa Y, Toyooka K, Matsuoka K, Jinbo T, Kimura T. 2007. Development of series of gateway binary vectors, pGWBs, for realizing efficient construction of fusion genes for plant transformation. Journal of Bioscience and Bioengineering 104, 34-41.

Navarro-Rodríguez M, Buesa JM, Rubio LM. 2019. Genetic and biochemical analysis of the Azotobacter vinelandii molybdenum storage protein. Frontiers in Microbiology 10, 579.

Nelson BK, Cai X, Nebenführ A. 2007. A multicolored set of in vivo organelle markers for co-localization studies in Arabidopsis and other plants. The Plant Journal 51, 1126-1136.

O'Hara GW. 2001. Nutritional constraints on root nodule bacteria affecting symbiotic nitrogen fixation: a review. Australian Journal of Experimental Agriculture 41, 417-433.

Rodríguez-Haas B, Finney L, Vogt S, González-Melendi P, Imperial J, González-Guerrero M. 2013. Iron distribution through the developmental stages of Medicago truncatula nodules. Metallomics 5, 1247-1253.

Rubio MC, James EK, Clemente MR, Bucciarelli B, Fedorova M, Vance CP, Becana M. 2004. Localization of superoxide dismutases and hydrogen peroxide in legume root nodules. Molecular Plant-Microbe Interactions 17, 1294-1305.

Rubio LM, Ludden PW. 2005. Maturation of nitrogenase: a biochemical puzzle. Journal of Bacteriology 187, 405-414.

Sauviac L, Niebel A, Boisson-Dernier A, Barker DG, de CarvalhoNiebel F. 2005. Transcript enrichment of Nod factor-elicited early nodule genes in purified root hair fractions of the model legume Medicago truncatula. Journal Experimental Botany 56, 2507-2513.

Senovilla M, Castro-Rodríguez R, Abreu I, Escudero V, Kryvoruchko I, Udvardi MK, Imperial J, González-Guerrero M. 2018. Medicago truncatula copper transporter 1 (MtCOPT1) delivers copper for symbiotic nitrogen fixation. New Phytologist 218, 696-709.

Tadege M, Wen J, He J, et al. 2008. Large-scale insertional mutagenesis using the Tnt1 retrotransposon in the model legume Medicago truncatula. The Plant Journal 54, 335-347.

Takanashi K, Yokosho K, Saeki K, Sugiyama A, Sato S, Tabata S, Ma JF, Yazaki K. 2013. LjMATE1: a citrate transporter responsible for iron supply to the nodule infection zone of Lotus japonicus. Plant \& Cell Physiology 54, 585-594.

Tejada-Jiménez M, Castro-Rodríguez R, Kryvoruchko I, Lucas MM, Udvardi M, Imperial J, González-Guerrero M. 2015. Medicago truncatula natural resistance-associated macrophage Protein1 is required for iron uptake by rhizobia-infected nodule cells. Plant Physiology 168, 258-272.

Tejada-Jiménez M, Gil-Díez P, León-Mediavilla J, Wen J, Mysore KS, Imperial J, González-Guerrero M. 2017. Medicago truncatula Molybdate transporter type 1 (MtMOT1.3) is a plasma membrane molybdenum transporter required for nitrogenase activity in root nodules under molybdenum deficiency. New Phytologist 216, 1223-1235.

Udvardi M, Poole PS. 2013. Transport and metabolism in legume-rhizobia symbioses. Annual Review of Plant Biology 64, 781-805.

Vernoud V, Journet EP, Barker DG. 1999. MtENOD20, a Nod factorinducible molecular marker for root cortical cell activation. Molecular PlantMicrobe Interactions 12, 604-614.

Walker EL, Waters BM. 2011. The role of transition metal homeostasis in plant seed development. Current Opinion in Plant Biology 14, 318-324.

Walton JH, Kontra-Kováts G, Green RT, Domonkos Á, Horváth B, Brear EM, Franceschetti M, Kaló P, Balk J. 2020. The Medicago truncatula Vacuolar Iron Transporter-Like proteins VTL4 and VTL8 deliver iron to symbiotic bacteria at different stages of the infection process. New Phytologist doi: 10.1111/nph.16735

Waters BM, Chu HH, Didonato RJ, Roberts LA, Eisley RB, Lahner B, Salt DE, Walker EL. 2006. Mutations in Arabidopsis yellow stripe-like1 and yellow stripe-like3 reveal their roles in metal ion homeostasis and loading of metal ions in seeds. Plant Physiology 141, 1446-1458.

Wood CC, Petrie JR, Shrestha P, Mansour MP, Nichols PD, Green AG, Singh SP. 2009. A leaf-based assay using interchangeable design principles to rapidly assemble multistep recombinant pathways. Plant Biotechnology Journal 7, 914-924.

Yeoman KH, Wisniewski-Dye F, Timony C, Stevens JB, deLuca NG, Downie JA, Johnston AWB. 2000. Analysis of the Rhizobium leguminosarum siderophore-uptake gene fhuA: differential expression in free-living bacteria and nitrogen-fixing bacteroids and distribution of an fhuA pseudogene in different strains. Microbiology 146, 829-837.

Yordem BK, Conte SS, Ma JF, Yokosho K, Vasques KA, Gopalsamy SN, Walker EL. 2011. Brachypodium distachyon as a new model system for understanding iron homeostasis in grasses: phylogenetic and expression analysis of Yellow Stripe-Like (YSL) transporters. Annals of Botany 108, 821-833.

Yruela I. 2013. Transition metals in plant photosynthesis. Metallomics 5, 1090-1109. 